\title{
Analysis of 56K genomes identifies the relationship between antibiotic and metal resistance co-Occurrence and the spread of multidrug-resistant non-typhoidal Salmonella
}

Gavin J Fenske ${ }^{1}$ and Joy Scaria ${ }^{1}$

${ }^{1}$ Department of Veterinary and Biomedical Sciences, South Dakota State University, Brookings, South Dakota, USA, 57007

\begin{abstract}
Salmonella enterica is common foodborne pathogen that generates both enteric and systemic infections in hosts. Antibiotic resistance is common is certain serovars of the pathogen and of great concern to public health. Recent reports have documented the co-occurrence of metal resistance with antibiotic resistance in one serovar of $S$. enterica. Therefore, we sought to identify possible co-occurrence in a large genomic dataset. Genome assemblies of 56,348 strains of $S$. enterica comprising 20 major serovars were downloaded from NCBI. The downloaded assemblies were quality controlled and in silico serotyped to ensure consistency and avoid improper annotation from public databases. Metal and antibiotic resistance genes were identified in the genomes as well as plasmid replicons. Co-current genes were identified by constructing a co-occurrence matrix and group the genes using k-means clustering. Three groups of cooccurrent genes were identified using k-means clustering. Group 1 was comprised of the pco and sil operons that confer resistance to copper and silver respectively. Group 1 was distributed across four serovars. Group 2 contained the majority of the genes and little to no co-occurrence was observed. Metal and antibiotic co-occurrence was identified in group 3 that contained genes conferring resistance to: arsenic, mercury, beta-lactams, sulfonamides, and tetracyclines. Group 3 genes were also associated with an IncQ1 class plasmid replicon. Metal and antibiotic cooccurrence was isolated to one clade of S. enterica I 4,[5],12:i:.
\end{abstract}

Correspondence: joy.scaria@sdstate.edu

Keywords: Antibiotic Resistance, Metal Resistance, Co-Occurrence, Salmonella enterica, Genomics

\section{INTRODUCTION}

Antibiotic resistant Salmonella enterica subspecies enterica is classified as a serious threat to public health by the Centers for Disease Control and Prevention in the United States [1]. S. enterica can rapidly disseminate antimicrobial resistance (AMR) genes horizontally, given the pathogen infects a wide range of hosts. Such a broad host range presents a unique challenge to public health. It has been demonstrated that sub-clinical doses of veterinary antibiotics in feed can promote the acquisition of resistance genes to clinically relevant antibiotics [2]. In this way, S. enterica acts as an antibiotic resistance bridge between agricultural and clinical settings. To combat the spread of AMR in bacteria, metals such as copper and zinc have been proposed and adopted by some producers as an alternative to antibiotics [3, 4]. One supplement, copper sulphate, has been proposed as a growth promoter in swine feed since at least 1961 [5]. The 
interest in metals as alternatives to antibiotics as growth promotors is obvious: metals are not antibiotics, thus curbing the spread of antibiotic resistance, copper is relatively inexpensive, and the growth promoting benefits, namely feed conversion efficiency, are retained when using the metal. When the European Union decided to ban the use of antibiotics as growth promoters in animal feed in 2006, many pig producers looked to copper sulphate as an alternative [6]. However, it has been shown that pharmacological doses of copper sulphate in pig feed increases antibiotic resistance in Escherichia coli [7]. Conjugative plasmids harboring resistance to macrolides, glycopeptides, and copper sulphate have been isolated from strains of Enterococcus faecium [8]. When metal and antibiotic resistance genes are constituents in the same linkage groups (plasmids, transposons, phages, etc) metals will co-select for antibiotic resistance genes [9-11]. It is becoming evident that metals are not a suitable alternative to antibiotics in agricultural settings and provide that same selective pressures as the antibiotics they are intended to replace [12].

Co-occurrence of metal and antibiotic resistance genes in S. enterica have been documented in S. enterica I 4,[5],12:i:- [13-16] and it has been suggested that copper resistance has allowed for expansion of the once rare serovar [16]. Given S. enterica's unique ability to act as a zoonotic disseminator of AMR and metal resistance genes, we sought to identify co-occurrence between metal and antibiotic resistance genes in a query set of $>56,000$ genomes comprising 20 major serovars. We specifically focused upon metal resistance genes that are plasmid bound to focus our analysis on genes that could be horizontally transmitted.

\section{RESULTS}

\section{Broad screen for metal and antibiotic co-occurrence in S. enterica}

The aim of our study was to identify co-occurrence between metal and antibiotic resistance genes in S. enterica with the potential for recombination (plasmid bound). To begin the screening, approximately 81,000 genome assemblies were downloaded from NCBI, quality checked, and in silico serotyped. The twenty most abundant serotypes, all containing greater than or nearly 1,000 assemblies (S. enterica Javiana, $\mathrm{n}=995$ ), were taken for further analysis (table 1).

Table 1. List of serovars and number of genome assemblies, median assembly length, and date range of assemblies used to identify co-occurrence of metal and antibiotic resistance.

\begin{tabular}{|l|c|c|c|}
\hline Serotype & $\begin{array}{c}\text { Number of } \\
\text { genomes }\end{array}$ & $\begin{array}{c}\text { Median Length } \\
(\mathrm{Mb})\end{array}$ & Date Range \\
\hline Enteritidis & 11325 & 4.69 & $1950-2019$ \\
\hline Typhimurium & 8561 & 4.91 & $1958-2019$ \\
\hline Kentucky & 4131 & 4.92 & $1972-2019$ \\
\hline Infantis & 3866 & 4.94 & $1971-2019$ \\
\hline I 4,[5],12:i:- & 3657 & 4.95 & $1985-2019$ \\
\hline Newport & 3646 & 4.76 & $1975-2019$ \\
\hline Typhi & 2736 & 4.74 & $1958-2019$ \\
\hline Heidelberg & 2454 & 4.89 & $1979-2019$ \\
\hline Montevideo & 1841 & 4.65 & $1997-2019$ \\
\hline Agona & 1718 & 4.82 & $1952-2019$ \\
\hline
\end{tabular}




\begin{tabular}{|l|c|c|c|}
\hline Muenchen & 1641 & 4.79 & $1987-2019$ \\
\hline Saintpaul & 1570 & 4.79 & $1974-2019$ \\
\hline Anatum & 1560 & 4.73 & $1993-2019$ \\
\hline Senftenberg & 1329 & 4.81 & $2001-2019$ \\
\hline Schwarzengrund & 1130 & 4.81 & $2000-2019$ \\
\hline Mbandaka & 1083 & 4.75 & $2000-2019$ \\
\hline Braenderup & 1044 & 4.69 & $1999-2019$ \\
\hline Hadar & 1038 & 4.74 & $1988-2019$ \\
\hline Derby & 1023 & 4.87 & $1986-2019$ \\
\hline Javiana & 995 & 4.61 & $1995-2019$ \\
\hline
\end{tabular}

The final dataset totaled 56,348 S. enterica assemblies. Metal resistance, antibiotic resistance, and plasmid replicons were predicted from each assembly. To gain understanding into the temporal distribution of metal and antibiotic resistance, the mean number of antibiotic and metal resistance genes were plotted against year of collection as shown in Figure 1. Both resistance types show serovar specific trends. Many of the serovars show no trend between mean number of antibiotic and metal resistance genes over time. However, three serovars, S. enterica Kentucky, S. enterica I 4,[5],12:i:-, and S. enterica Infantis, have increasing antibiotic and metal resistance genes over time. The trend is most evident in S. enterica I 4,[5],12:i:-, with the mean number of metal resistance genes increasing nearly 3 fold in 15 years. S. enterica Schwarzengrund and S. enterica Senftenberg showed constant metal resistance with no trend observed to antibiotic resistance. S. enterica Enteritidis, the most abundant serovar, showed almost no antibiotic nor metal resistance homologues.

\section{Co-occurrence of metal and antibiotic resistance}

Co-occurrence between plasmid replicons (included as the focus was to identify possible recombinant resistance), metal resistance genes, and antibiotic resistance genes was identified by applying k-means clustering to a co-occurrence matrix (see methods). Three groups of cooccurrent genes were identified (figure 2A). To better visualize the groups identified by k-means clustering, the co-occurrence network was plotted, and groups were colored respective to figure 


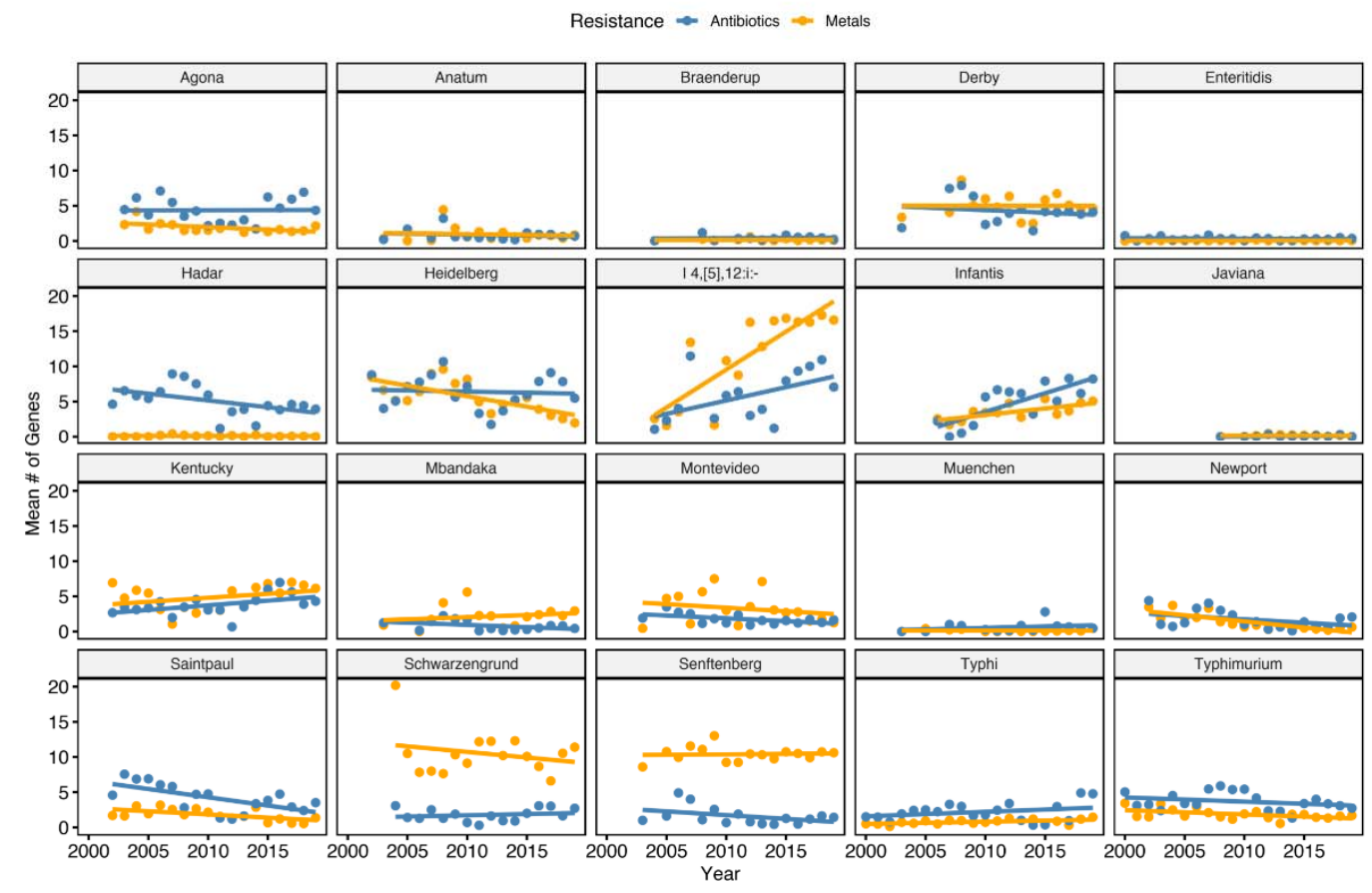

Figure 1. Temporal distribution of antibiotic and metal resistance genes by S. enterica serovar. The mean number of antibiotic and resistance homologues identified in each serovar is plotted against the date of collection. Only dates that contained $n>10$ genomes were considered to control for outliers.

A

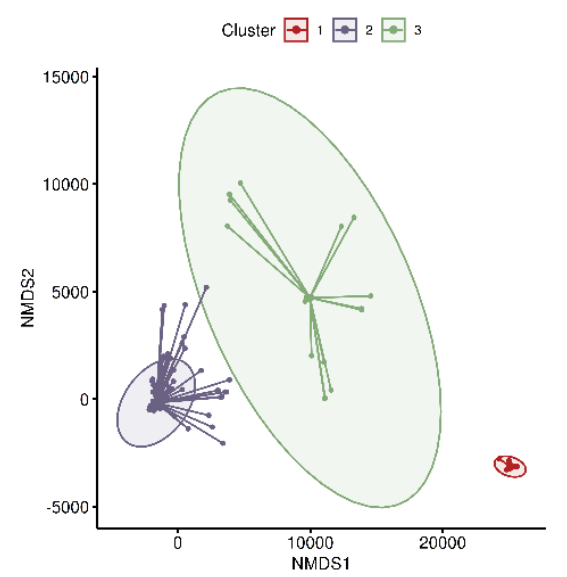

B

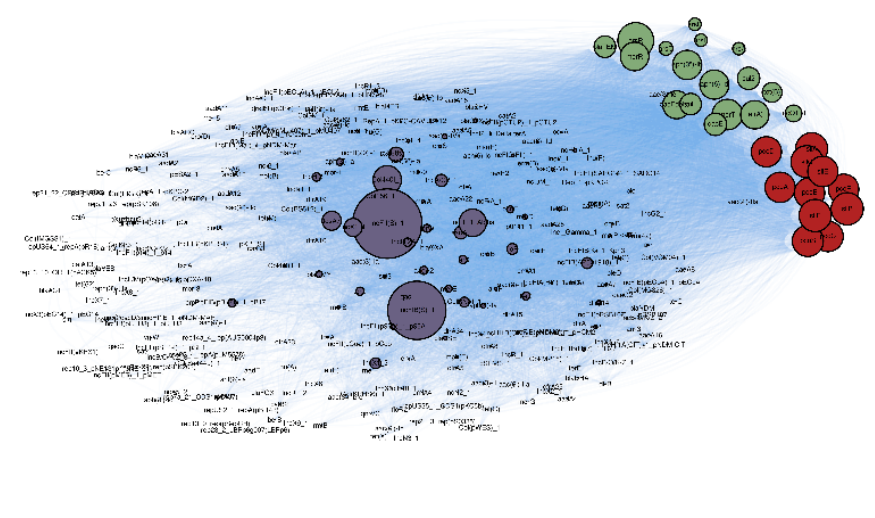

Figure 2. Co-occurrent gene clusters identified in S. enterica genomes. (A) Non-metric Multidimensional Scaling plot of a co-occurrence matrix generated from plasmid replicons, antibiotic and metal resistance homologues. K-means clustering was used to group genes. An elbow plot was used to pick the optimal number of clusters. Clusters are drawn as a star plot with lines connecting each gene to the center of the cluster. Gaussian distributions were drawn around each cluster. (B) Network representation of co-occurrence network. Nodes are scaled to edge weight (number of connections) and are colored consistent to A.

$2 \mathrm{~A}$ (figure 2B). Group 1 was the smallest cluster set containing only 11 genes comprising the pco and sil operons. Said operons confer resistance to copper and silver respectively. Group 2 
represents most plasmid replicons, metal resistance genes, and antibiotic resistance genes. Little co-occurrence is observed in group 2 save for two plasmid replicons (IncFIB(S)_1 and IncFII(S)_1). The small node sizes of other constituents in group 2 suggests that gene turnover may be high in the associated plasmids. The majority of antibiotic and metal resistance genes are not co-occurrent in the dataset of $S$. enterica genomes. However, co-occurrence was identified in group 3. Homologues in group 3 confer resistance to metals arsenic and mercury, antibiotic classes of beta-lactams, sulfonamides, tetracyclines, and contains one plasmid replicon: IncQ1_1. The top down approach and simple k-means clustering was able to identify that co-occurrence between metal and antibiotic resistance is occurring in some genomes of S. enterica. However, it also showed that the effect is only occurring in a small subset of genes, and that most are not carried together. The full list of homologues in groups 1 and 3 are presented in table 2 .

Table 2. Major groups of co-occurrent genes identified by k-means clustering.

\begin{tabular}{|c|c|c|c|}
\hline Cluster & Gene & Class & Subclass \\
\hline \multirow{11}{*}{1} & pcoA & Copper & Copper \\
\hline & pcoB & Copper & Copper \\
\hline & pcoC & Copper & Copper \\
\hline & pcoD & Copper & Copper \\
\hline & pcoR & Copper & Copper \\
\hline & pcoS & Copper & Copper \\
\hline & silA & Silver & Silver \\
\hline & silB & Silver & Silver \\
\hline & silC & Silver & Silver \\
\hline & silE & Silver & Silver \\
\hline & silF & Silver & Silver \\
\hline \multirow{17}{*}{3} & aph(3")-Ib & Aminoglycoside & Streptomycin \\
\hline & aph(6)-Id & Aminoglycoside & Streptomycin \\
\hline & $\operatorname{ars} \mathrm{A}$ & Arsenic & Arsenite \\
\hline & $\operatorname{arsB}$ & Arsenic & Arsenite \\
\hline & $\operatorname{arsC}$ & Arsenic & Arsenate \\
\hline & $\operatorname{arsD}$ & Arsenic & Arsenite \\
\hline & $\operatorname{arsR}$ & Arsenic & Arsenite \\
\hline & blaTEM & Beta-Lactam & Beta-Lactam \\
\hline & IncQ1_1 & Plasmid Replicon & NA \\
\hline & merR & Mercury & Mercury \\
\hline & merT & Mercury & Mercury \\
\hline & qacE & Quaternary Ammonium & Quaternary Ammonium \\
\hline & qacEdelta1 & Quaternary Ammonium & Quaternary Ammonium \\
\hline & sul1 & Sulfonamide & Sulfonamide \\
\hline & sul2 & Sulfonamide & Sulfonamide \\
\hline & $\operatorname{tet}(\mathrm{A})$ & Tetracycline & Tetracycline \\
\hline & $\operatorname{tet}(\mathrm{B})$ & Tetracycline & Tetracycline \\
\hline
\end{tabular}




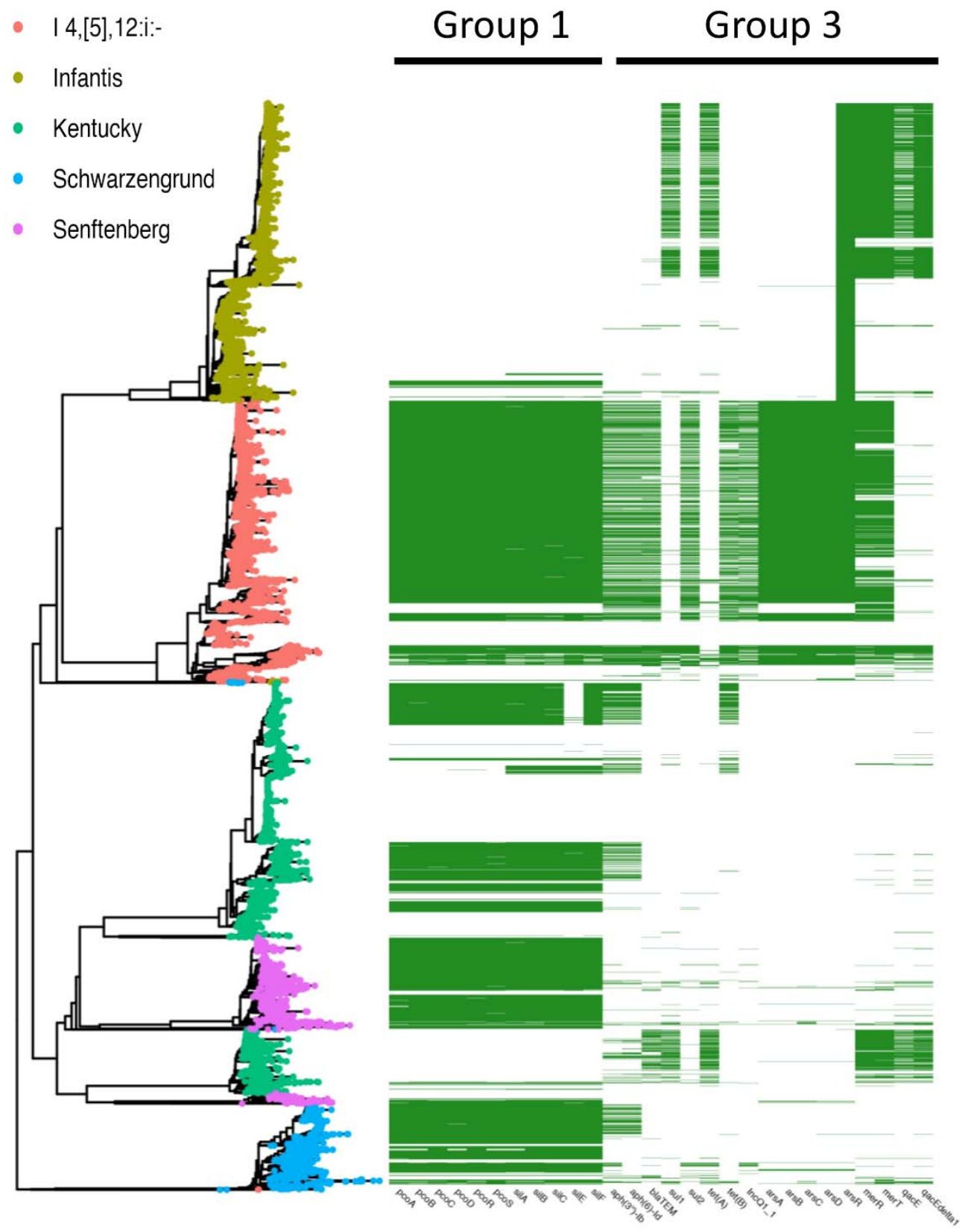

Figure 3. Distribution of group 1 and 3 genes among $S$. enterica I 4,[5],12:i:-, S. enterica Infantis, S. enterica Kentucky, S. enterica Schwarzengrund, and S. enterica Senftenberg. A prescence-absence matrix of group 1 and 3 genes is plotted against a neighbor-joining tree constructed from pairwise distance matrix. Group 1 (pco and sil operons) is found in S. enterica Kentucky, S. enterica Schwarzengrund, and S. enterica Senftenberg but is mostly absent from S. enterica Infantis. Group 3, which contains metal and antibiotic resistance genes, is almost exclusive to $S$. enterica I 4,[5],12:i:-. 
To better visualize the distribution of group 1 and 3 gene clusters (moderate to high co-occurrent genes), a presence-absence matrix of group 1 and 3 genes was plotted against the phylogeny of $S$. enterica I 4,[5],12:i:-, S. enterica Infantis, S. enterica Kentucky, S. enterica Schwarzengrund, and S. enterica Senftenberg (serovars that showed trends in figure 1) as shown in figure 3. Group 1 (sil and pco ) genes are broadly distributed among S. enterica I 4,[5],12:i:-, S. enterica Kentucky, S. enterica Schwarzengrund, and S. enterica Senftenberg, but are largely absent from S. enterica Infantis. However, group 1 genes only confer resistance to the metals silver and copper, and not antibiotics. Only S. enterica I 4,[5],12:i:- assemblies consistently contained loci from group 3, the co-occurrent group of metal and antibiotic resistance genes. Therefore, we decided to focus the study upon $S$. enterica I 4,[5],12:i:- and the possible mechanisms of the co-occurrence. Recent research has proposed the addition of metals, namely silver and copper, to medical devices, food pack

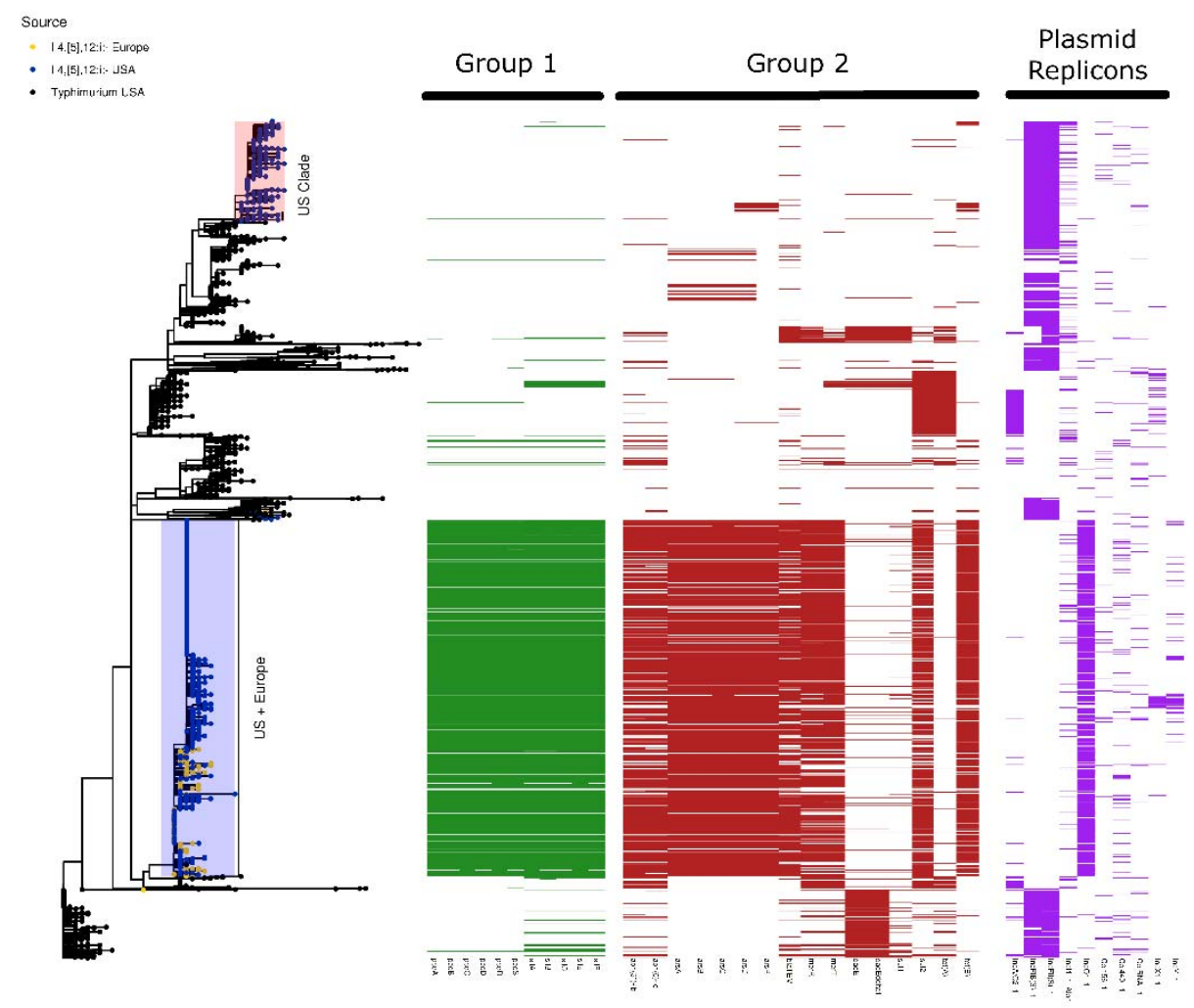

Figure 4. Distribution of group 1, group3, and major plasmid replicons in S. enterica I 4,[5],12:i:-. A presence-absence matrix for each gene group is plotted against a maximumlikelihood tree of S. enterica Typhimurium and S. enterica I 4,[5], 12:i:- rooted a reference S. enterica Typhimurium DT104 assembly. Two major clades of S. enterica I 4,[5],12:i:- are observed, however only the clade containing both US and European isolates contains both group 1 and group 3 genes as well as the IncQ1_1 plasmid replicon.

\section{S. enterica I 4,[5],12:i:-}

S. enterica I 4,[5],12:i:- was the only serovar to contain group 3 genes in a substantive manner. The initial dataset was compiled from assembled genomes in order to facilitate speed. However, the differing assembly mechanisms and lack of SNP based phylogeny limits the accuracy. To 
remedy this, 1,455 sequencing reads of US S. enterica I 4,[5],12:i:-, 1,447 sequencing reads of US S. enterica Typhimurium and 329 sequencing reads of European S. enterica I 4,[5],12:i:were compiled into a SNP phylogeny against a reference S. typhimurium DT104 assembly. The addition of the new data set allows for a validation step against the initial screening. Plasmid replicons, metal and antibiotic resistance were annotated in the second dataset exactly following the protocol for the large query set. A presence-absence matrix describing group 1, 3, and major plasmid replicons is plotted against the phylogeny of S. enterica I 4,[5],12:i:- and S. enterica Typhimurium in figure 4.

Confirming the initial screening, group 1 and 3 genes were carried in S. enterica I 4,[5],12:i:- but were mostly absent from S. Typhimurium. Two major clades of S. enterica I 4,[5],12:i:- are observed: one comprising solely of US isolates and a mixed clade of US and European genomes. Notably, the mixed clade of US and European isolates contain both group 1 and 3 as well as the IncQ1_1 plasmid replicon. The S. enterica I 4,[5],12:i:- clade comprised solely of US genomes did not contain group 1 and 3 genes. To this end, the co-occurrence of metal and antibiotic resistance is not widespread throughout $\mathrm{S}$. enterica, but rather is localized to one clade of $\mathrm{S}$. enterica I 4,[5],12:i:- . The metadata for the detailed analysis of S. enterica I 4,[5],12:i:- and S. enterica Typhimurium is provided in supplemental table 5.

\section{DISCUSSION}

In this work, we identified co-occurrence of metal and antibiotics in S. enterica I 4,[5],12:i:- by screening a broad set of 20 serovars totaling more than 56,000 genomes. The topdown approach allows for a non-biased and comprehensive approach compared to starting with metal resistant isolates and working upwards towards the serovar level. Ultimately, our hypothesis was incorrect as co-occurrence between metal and antibiotic resistance is not widespread in S. enterica. Rather, the phenomenon is limited to one clade of S. enterica I 4,[5],12:i:-. This result is interesting in that it highlights how the acquisition of resistance genes can lead to a rapid expansion of bacterial strains. S. enterica I 4,[5],12:i:- was rarely isolated in the 1990's but was the third most isolated serovar of S. enterica in the European Union in 2017 [29] and the fifth most isolated serovar in the US in 2015 [30]. It has been suggested that the introduction of copper sulfate into pig feed, and the resistance to copper harbored by $S$. enterica I 4,[5],12:i:- strains, may have contributed to the rapid increase in prevalence [16]. S. enterica I 4,[5],12:i:- provides a meaningful example of the link of agricultural processes and human health. While many of the isolates did not contain the co-occurrence between antibiotics and metals we sought to identify, copper and silver resistance was identified in S. enterica Kentucky, S. enterica Schwarzengrund, and S. enterica Senftenberg.

The pco and sil operons are associated in S. enterica with Salmonella Genomic Island 4 (SGI-4). SGI-4 is a chromosomal island containing the pco and sil operons that may be excised from the chromosome by mitomycin $\mathrm{C}$, and oxygen tension related stress [13]. SGI-4 was first designated in 2016 [16](see addendum) and is largely associated with S. enterica I 4,[5],12:i:[13]. Indeed it has been stated that SGI-4 has only been discovered in I 4,[5],12:i:- [31]. Our results indicate the genomic island may be more widespread than believed. As metal use increases in medical and agricultural settings, the opportunity exists for metal resistant strains to rapidly expand in prevalence as has been witnessed with $S$. enterica I 4,[5],12:i:-. Indeed, a burn ward identified S. enterica Senftenberg as a causative agent of an outbreak in severe burn patients [32]. The isolated strains were resistant to the silver sulfadiazine that was applied daily 
to the burn patients. Another concern is the increasing use of copper sulphate as a growth promoter in chickens $[33,34]$. S. enterica Schwarzengrund is commonly isolated from chickens and global outbreaks of multi-drug resistant strains have occurred [35]. If metal use in poultry agriculture continues, S. enterica Schwarzengrund outbreaks have the potential to increase in the coming years.

The serovar specific nature of group 3 genes (metal and antibiotic co-occurrence) is consistent with earlier reports $[14,36,37]$. Previous work has demonstrated that a clade $S$. enterica I 4,[5],12:i:- circulating the US is descendent from multi-drug resistant strains in Europe [38]. The inclusion of the IncQ1_1 plasmid replicon reinforces the claim as the gene was the most identified plasmid replicon of S. enterica I 4,[5],12:i:- in Italian isolates [14]. A plasmid harboring resistance to metals and antibiotics has been documented in an Australian S. enterica I 4,[5],12:i:- isolated from pig feces [15]. However, the plasmid was large ( $275 \mathrm{~kb})$ and annotated as a IncHI 2 class plasmid, not an IncQ class as we report here. Additionally, it was found that the plasmid was able to conjugate to other species [15]. IncQ plasmids are unable to self-transfer and rely upon "helper plasmids" for transmission between bacterial cells [39]. The lack of other plasmid replicons in large numbers in the mixed US and European clade of $S$. enterica I 4,[5],12:i:-, and isolation of group 3 genes in the clade, suggests the plasmid and group 3 genes are being transmitted vertically rather than horizontally. Furthermore, IncQ class plasmids are evolutionary stable with one study documenting the presence of the plasmid in the environment over a 30 year period [40]. Taken together, we propose that the method of metal and antibiotic co-occurrence in the US and European monophasic strains studied here differs from the large IncHI2 plasmid isolated from an Australian strain of S. enterica I 4,[5],12:i:- . Complicating the narrative is the difficulty in accurately constructing plasmid contigs from shortread sequencing [41]. Isolating plasmids from a large number of $S$. enterica I 4,[5],12:i:containing the IncQ1_1 and group 3 genes and sequencing with a long-read platform such as PacBio is needed to confirm our results.

\section{MATERIALS AND METHODS}

\section{Salmonella enterica genome assembly acquisition}

Genome assemblies and GenBank files for S. enterica isolates were downloaded from Genome on NCBI (ftp.ncbi.nih.gov::genomes/all/GCA/, October 10, 2019). Resultant GenBank files were parsed and only assemblies containing a collection date were included for further analysis. Downloaded assemblies were removed from the data set if the contig number was greater than 300. Genome assemblies were in silico serotyped using SISTR [17]. This was done to ensure consistent serovar annotation and limit the impact of improper or mislabeled serotypes. The top 20 serovars in the dataset were used for co-occurrence identification. 20 serovars were chosen as each contained greater than 1,000 genomes, save for $\mathrm{S}$. enterica Javiana, which contained $\mathrm{n}=$ 995.

\section{Identification of plasmid replicons, metal and antibiotic resistance homologues}

Plasmid replicons were identified in genome assemblies using the tool Abricate [18] against the PlasmidFinder [19] database. Hits were defined as sequences with $>=90 \%$ percent identity and $>=60 \%$ query coverage. 
Open-reading frames (ORF) in assemblies were predicted using Prodigal [20]. Metal resistance homologues were identified in the resultant ORF files using GhostZ [21] to compare the ORF against the experimentally verified BacMet database (version 2.0) [22]. ORF were considered homologues when: e-value $<=1 \mathrm{E}-6$, percent identity $>=90 \%$ and percent coverage $>=60 \%$. As we aimed to identify metal resistance under possible recombination, only homologues marked "PLASMID" in the BacMet database were considered.

Antibiotic resistance homologues were identified in an analogous manner to the metal resistance genes, however, ORF were queried against the NCBI antimicrobial resistance database (BioProject: PRJNA313047) using the same parameters.

\section{Co-Occurrence identification}

Plasmid replicons, metal and antibiotic resistance homologues from each assembly were compiled into a binary matrix. A co-occurrence matrix was generated by taking the cross product of the original binary matrix. Simply put, if two genes are contained in the same assembly, they yield a value of 1 . If genes are contained across multiple assemblies together, the number will increase accordingly. To identify possible co-occurrence between genes, the co-occurrence matrix was subjected to k-means clustering using clusters. The optimal number of clusters was determined by constructing an "elbow plot" (the sum of square distances against k number of clusters). Matrix compilation and k-means clustering were conducted using R ( $\mathrm{R}$-core team). Igraph [23] was used to visualize the co-occurrence network. Nodes are scaled to edge weight number (larger nodes contain more co-occurrences).

\section{Phylogeny and S. enterica I,4,[5],12:i:- analysis}

To compile a phylogeny estimation of 56,348 S. enterica genomes, MASH [24] sketches were drawn from each sample (10,000 sketches, kmer size of 21) and a pairwise distance matrix was generated. A neighbor-joining tree was constructed from the distance matrix using QuickTree [25].

Reads of European and US S. enterica I,4,[5],12:i:- and US S. enterica typhimurium and were downloaded from SRA hosted at NCBI (https://www.ncbi.nlm.nih.gov/sra). Resultant reads were mapped reads against a $S$. enterica Typhimurium DT104 NCTC 13348 reference strain downloaded from https://www.sanger.ac.uk/resources/downloads/bacteria/salmonella.html. Mapping and variant calling was conducted using Snippy [26] and only core variants were considered. A maximum likelihood from the SNP alignment was generated using IQTree [27] with a GTR+G4 substitution model and 1000 bootstraps. All phylogenies were visualized using the $\mathrm{R}$ package ggtree [28].

Reads were assembled into contigs using the package Shovill (https://github.com/tseemann/shovill) with a minimum contig length of 200 base pairs. Prodigal was used to predict ORF and plamid replicons, antibiotic and metal resistance genes were predicted in the same manner as the genomes of the large test set. 
Funding: This work was funded by the US Food and Drug Administration (FDA) grant 1U19FD007117 and United States Department of Agriculture (USDA) grants SD00H702-20 and SD00R646-18.

Data Availability Statement: Salmonella genome data used in this analysis is public data already deposited in NCBI SRA. All salmonella genomes available in NCBI SRA up to October 10, 2019 was used for the analysis.

Acknowledgments: Authors gratefully thank Dr. Marc W. Allard, and Dr. Ruth Timme, FDA CFSAN ORS and the GenomeTrakr Network labs which sequenced and submitted the Salmonella genome data to NCBI SRA which was used for the analysis in this manuscript.

Conflicts of Interest: The authors declare no conflict of interest

\section{REFERENCES:}

1. CDC. Antibiotic Resistance Threats in the United States. $\mathbf{2 0 1 9}$

2. Singh AK, Bhunia AK. Animal-Use Antibiotics Induce Cross-Resistance in Bacterial Pathogens to Human Therapeutic Antibiotics. Current Microbiology 2019; 76(10): 1112-7.

3. Liu Y, Espinosa CD, Abelilla JJ, et al. Non-antibiotic feed additives in diets for pigs: A review. Animal Nutrition 2018; 4(2): 113-25.

4. Carpenter CB, Woodworth JC, DeRouchey JM, et al. Effects of increasing copper from tri-basic copper chloride or a copper-methionine chelate on growth performance of nursery pigs 1,2 . Translational Animal Science 2018; 3(1): 369-76.

5. Lucas IAM, Livingstone RM, McDonald I. Copper sulphate as a growth stimulant for pigs: effect of level and purity. Animal Science 1961; 3(2): 111-9.

6. Slade RD, Kyriazakis I, Carroll SM, et al. Effect of rearing environment and dietary zinc oxide on the response of group-housed weaned pigs to enterotoxigenic Escherichia coli 0149 challenge. Animal 2011; 5(8): 1170-8.

7. Zhang Y, Zhou J, Dong Z, et al. Effect of Dietary Copper on Intestinal Microbiota and Antimicrobial Resistance Profiles of Escherichia coli in Weaned Piglets. Frontiers in microbiology 2019; 10: 2808.

8. Hasman H, Aarestrup FM. tcrB, a Gene Conferring Transferable Copper Resistance in Enterococcus faecium: Occurrence, Transferability, and Linkage to Macrolide and Glycopeptide Resistance. Antimicrobial Agents and Chemotherapy 2002; 46(5): 1410.

9. Baker-Austin C, Wright MS, Stepanauskas R, McArthur JV. Co-selection of antibiotic and metal resistance. Trends in Microbiology 2006; 14(4): 176-82.

10. Seiler C, Berendonk T. Heavy metal driven co-selection of antibiotic resistance in soil and water bodies impacted by agriculture and aquaculture. Frontiers in microbiology 2012; 3: 399.

11. Pal C, Bengtsson-Palme J, Kristiansson E, Larsson DGJ. Co-occurrence of resistance genes to antibiotics, biocides and metals reveals novel insights into their co-selection potential. BMC Genomics 2015; 16(1): 964.

12. Dickinson AW, Power A, Hansen MG, et al. Heavy metal pollution and co-selection for antibiotic resistance: A microbial palaeontology approach. Environ Int 2019; 132: 105117.

13. Branchu P, Charity OJ, Bawn M, et al. SGI-4 in Monophasic Salmonella Typhimurium ST34 Is a Novel ICE That Enhances Resistance to Copper. Frontiers in microbiology 2019; 10: 1118. 
14. Mastrorilli E, Pietrucci D, Barco L, et al. A Comparative Genomic Analysis Provides Novel Insights Into the Ecological Success of the Monophasic Salmonella Serovar 4,[5],12:i. Frontiers in microbiology 2018; 9: 715-.

15. Dyall-Smith ML, Liu Y, Billman-Jacobe H. Genome Sequence of an Australian Monophasic Salmonella enterica subsp. enterica Typhimurium Isolate (TW-Stm6) Carrying a Large Plasmid with Multiple Antimicrobial Resistance Genes. Genome Announc 2017; 5(33): e00793-17.

16. Liljana P, Alison EM, Manal A, et al. Microevolution of Monophasic Salmonella Typhimurium during Epidemic, United Kingdom, 2005-2010. Emerging Infectious Disease journal 2016; 22(4): 617.

17. Yoshida CE, Kruczkiewicz P, Laing CR, et al. The Salmonella In Silico Typing Resource (SISTR): An Open Web-Accessible Tool for Rapidly Typing and Subtyping Draft Salmonella Genome Assemblies. PLOS ONE 2016; 11(1): e0147101.

18. Seemann T. ABRicate. 0.8.0 ed. GitHub, 2018.

19. Carattoli A, Zankari E, García-Fernández A, et al. In silico detection and typing of plasmids using PlasmidFinder and plasmid multilocus sequence typing. Antimicrobial agents and chemotherapy 2014; 58(7): 3895-903.

20. Hyatt D, Chen G-L, Locascio PF, Land ML, Larimer FW, Hauser LJ. Prodigal: prokaryotic gene recognition and translation initiation site identification. BMC bioinformatics 2010; 11: 119-.

21. Suzuki S, Kakuta M, Ishida T, Akiyama Y. Faster sequence homology searches by clustering subsequences. Bioinformatics 2014; 31(8): 1183-90.

22. Pal C, Bengtsson-Palme J, Rensing C, Kristiansson E, Larsson DGJ. BacMet: antibacterial biocide and metal resistance genes database. Nucleic acids research 2014; 42(Database issue): D737D43.

23. Csardi G, Nepusz T. The Igraph Software Package for Complex Network Research. InterJournal 2005; Complex Systems: 1695.

24. Ondov BD, Treangen TJ, Melsted P, et al. Mash: fast genome and metagenome distance estimation using MinHash. Genome Biology 2016; 17(1): 132.

25. Howe K, Bateman A, Durbin R. QuickTree: building huge Neighbour-Joining trees of protein sequences. Bioinformatics 2002; 18(11): 1546-7.

26. Seemann T. snippy: fast bacterial variant calling from NGS reads. https://github.com/tseemann/snippy, 2015.

27. Nguyen L-T, Schmidt HA, von Haeseler A, Minh BQ. IQ-TREE: a fast and effective stochastic algorithm for estimating maximum-likelihood phylogenies. Mol Biol Evol 2015; 32(1): 268-74.

28. Yu G, Smith DK, Zhu H, Guan Y, Lam TT-Y. ggtree: an $r$ package for visualization and annotation of phylogenetic trees with their covariates and other associated data. Methods in Ecology and Evolution 2017; 8(1): 28-36.

29. European Food Safety A, European Centre for Disease P, Control. The European Union summary report on trends and sources of zoonoses, zoonotic agents and food-borne outbreaks in 2017. EFSA Journal 2018; 16(12): e05500.

30. CDC. Foodborne Diseases Active Surveillance Network (FoodNet): FoodNet 2015 Surveillance Report (Final Data). 2017.

31. Branchu P, Bawn M, Kingsley RA. Genome Variation and Molecular Epidemiology of Salmonella enterica Serovar Typhimurium Pathovariants. Infection and Immunity 2018; 86(8): e00079-18.

32. Nair D, Gupta N, Kabra S, Ahuja RB, Prakash SK. Salmonella senftenberg: a new pathogen in the burns ward. Burns 1999; 25(8): 723-7.

33. Scott A, Vadalasetty KP, Łukasiewicz M, et al. Effect of different levels of copper nanoparticles and copper sulphate on performance, metabolism and blood biochemical profiles in broiler chicken. Journal of Animal Physiology and Animal Nutrition 2018; 102(1): e364-e73. 
34. ŚWiĄTkiewicz S, Arczewska-WŁOsek A, JÓZefiak D. The efficacy of organic minerals in poultry nutrition: review and implications of recent studies. World's Poultry Science Journal 2014; 70(3): 475-86.

35. Aarestrup FM, Hendriksen RS, Lockett J, et al. International spread of multidrug-resistant Salmonella Schwarzengrund in food products. Emerg Infect Dis 2007; 13(5): 726-31.

36. Mourão J, Marçal S, Ramos $P$, et al. Tolerance to multiple metal stressors in emerging nontyphoidal MDR Salmonella serotypes: a relevant role for copper in anaerobic conditions. Journal of Antimicrobial Chemotherapy 2016; 71(8): 2147-57.

37. Billman-Jacobe H, Liu Y, Haites R, et al. pSTM6-275, a Conjugative IncHI2 Plasmid of Salmonella enterica That Confers Antibiotic and Heavy-Metal Resistance under Changing Physiological Conditions. Antimicrobial Agents and Chemotherapy 2018; 62(5): e02357-17.

38. Elnekave E, Hong S, Mather AE, et al. Salmonella enterica Serotype 4,[5],12:i:- in Swine in the United States Midwest: An Emerging Multidrug-Resistant Clade. Clinical Infectious Diseases 2017; 66(6): 877-85.

39. Rawlings DE, Tietze E. Comparative biology of IncQ and IncQ-like plasmids. Microbiol Mol Biol Rev 2001; 65(4): 481-96.

40. Yau S, Liu X, Djordjevic SP, Hall RM. RSF1010-Like Plasmids in Australian Salmonella enterica Serovar Typhimurium and Origin of Their sul2-strA-strB Antibiotic Resistance Gene Cluster. Microbial Drug Resistance 2010; 16(4): 249-52.

41. Arredondo-Alonso S, Willems RJ, van Schaik W, Schurch AC. On the (im)possibility of reconstructing plasmids from whole-genome short-read sequencing data. Microb Genom 2017; 3(10): e000128. 